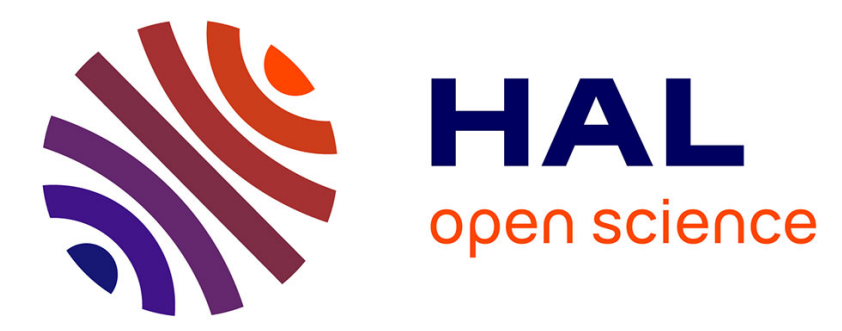

\title{
Elastic properties of AlAs-like and InSb-like strained interfaces in [InAs/AlSb] heterostructures
}

Yann Claveau, Maxime Vallet, Hao Tang, Nicolas Combe, Anne Ponchet

\section{To cite this version:}

Yann Claveau, Maxime Vallet, Hao Tang, Nicolas Combe, Anne Ponchet. Elastic properties of AlAslike and InSb-like strained interfaces in [InAs/AlSb] heterostructures. Applied Physics Letters, 2016, 109, pp.41903 - 211908. 10.1063/1.4959843 . hal-01743639

\section{HAL Id: hal-01743639 \\ https://hal.science/hal-01743639}

Submitted on 26 Mar 2018

HAL is a multi-disciplinary open access archive for the deposit and dissemination of scientific research documents, whether they are published or not. The documents may come from teaching and research institutions in France or abroad, or from public or private research centers.
L'archive ouverte pluridisciplinaire HAL, est destinée au dépôt et à la diffusion de documents scientifiques de niveau recherche, publiés ou non, émanant des établissements d'enseignement et de recherche français ou étrangers, des laboratoires publics ou privés. 


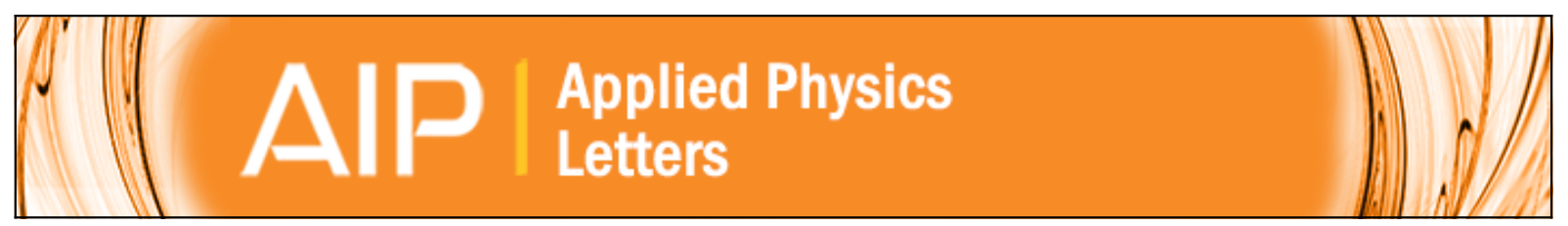

\section{Elastic properties of AIAs-like and InSb-like strained interfaces in [InAs/AISb] heterostructures}

Y. Claveau, M. Vallet, H. Tang, N. Combe, and A. Ponchet

Citation: Applied Physics Letters 109, 041903 (2016); doi: 10.1063/1.4959843

View online: http://dx.doi.org/10.1063/1.4959843

View Table of Contents: http://scitation.aip.org/content/aip/journal/apl/109/4?ver=pdfcov

Published by the AIP Publishing

\section{Articles you may be interested in}

Highly strained AlAs-type interfaces in InAs/AISb heterostructures

Appl. Phys. Lett. 108, 211908 (2016); 10.1063/1.4952951

Elastic strains at interfaces in InAs/AISb multilayer structures for quantum cascade lasers

Appl. Phys. Lett. 104, 031907 (2014); 10.1063/1.4863035

Anisotropic transport properties in InAs/AISb heterostructures

Appl. Phys. Lett. 97, 243510 (2010); 10.1063/1.3527971

Interface tuning of the InAs/AISb heterostructure-based quantum wells

J. Vac. Sci. Technol. B 18, 2279 (2000); 10.1116/1.1303810

Localized interface states and the optical spectra of AISb/lnAs heterostructures

J. Vac. Sci. Technol. B 16, 1794 (1998); 10.1116/1.590232

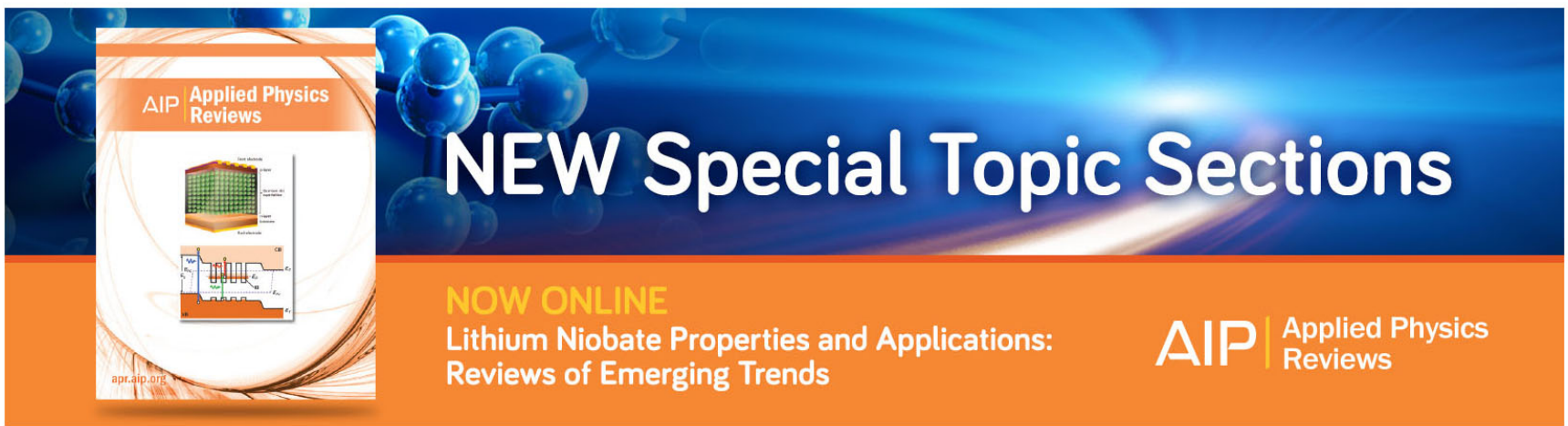




\title{
Elastic properties of AIAs-like and InSb-like strained interfaces in [InAs/AISb] heterostructures
}

\author{
Y. Claveau, M. Vallet, H. Tang, N. Combe, and A. Ponchet ${ }^{\mathrm{a})}$ \\ CEMES CNRS UPR8011, Université de Toulouse, 29 rue Jeanne Marvig, 31055 Toulouse, France
}

(Received 7 June 2016; accepted 12 July 2016; published online 27 July 2016)

\begin{abstract}
Elastic properties of [InAs/AlSb] heterostructures coherently grown on a (001) InAs substrate are investigated by the density functional theory and compared to the prediction of the linear elasticity theory. The stress-strain curves of the four involved binaries (InAs, AlAs, AlSb, and InSb) are first studied: a significant deviation to the linear elasticity theory is observed for strain above $2.5 \%$ (in absolute value). Nevertheless, the relationship between the out-of-plane and in-plane strains is in a good agreement with the prediction of the linear elasticity theory. In the heterostructures, highly strained perfect AlAs-like and InSb-like interfaces are examined. The interfacial strains calculated using the density functional theory are in a surprisingly good agreement with the prediction of the linear elasticity theory. The reduction of the layer thickness to the thinnest possible InAs or AlSb layers while keeping perfect interfaces does not change these conclusions. Published by AIP Publishing.

[http://dx.doi.org/10.1063/1.4959843]
\end{abstract}

In epitaxial nanostructures, while elastic strains can be much higher than in bulk materials, the elastic properties are often studied in the frame of the linear elasticity theory, which relates linearly the strain and stress tensors (generalized Hooke's law). ${ }^{1}$ For instance, the chemical composition is frequently deduced from a strain analysis (from atomic resolved transmission electron microscopy (TEM) images) relying on the linear elasticity theory. However, it presents two well-known limits: (i) beyond a certain level of strain, the stress does no more evolve linearly with the strain and (ii) it is a continuum theory which in principle does not apply at the atomic scale. To experimentally address these limits, the strain and the stress should be recovered independently, which is difficult in nanostructures.

Here, we investigate the [InAs/AlSb] system grown on a (001) InAs substrate that presents a large offset of the conduction band, favorable for devices like quantum cascade lasers (QCLs) exploiting intersubband transitions. ${ }^{2}$ Due to the lack of common atoms between InAs and AlSb, the interfaces necessarily involve $\mathrm{Al}-\mathrm{As}$ or In-Sb bonds also associated with specific electronic interfacial properties. ${ }^{3}$ Besides, it is also a good candidate to simultaneously investigate the two mentioned limits of the linear elasticity theory. Indeed, in their bulk state, AlAs and InSb present strong misfits of $-6.6 \%$ and $6.9 \%$ with InAs, respectively. Experimentally, spontaneously formed interfaces are not perfect and extend over 2 or 3 monolayers, but they could reach the same level of strain as expected for a perfect tensile Al-As type interface. ${ }^{4}$ Using a modified growth procedure, interfaces with a high compressive stress were also achieved. ${ }^{5}$ High and localized strains also exist in various mismatched heterostructures, thanks to the same interfacial configuration as here $\left([\mathrm{InAs} / \mathrm{GaSb}]^{6}\right.$ and $\left.[\mathrm{ZnTe} / \mathrm{CdSe}]^{7}\right)$ or to a nanowire geometry ${ }^{8-10}$ for instance.

In this article, the elastic properties of [InAs/AlSb] heterostructures are theoretically investigated with a special

\footnotetext{
${ }^{\text {a) }}$ Author to whom correspondence should be addressed. Electronic mail: anne.ponchet@cemes.fr
}

emphasis on the perfect interfaces of AlAs- or InSb-type. Interfaces were studied using atomistic modeling based on the Density Functional Theory (DFT). DFT studies have already shown their reliability to study elastic properties and evidenced the non-linear elastic response of InAs, even at moderate biaxial strain. ${ }^{11}$ Below, after giving the simulation details we provide the elastic responses to biaxial strain from $-7 \%$ to $7 \%$ of the binary materials involved here (InAs, $\mathrm{AlSb}, \mathrm{AlAs}$, and InSb). We then investigate the interfaces of [InAs/AlSb] heterostructures and systematically compare DFT results with those of the linear elasticity. As the QCL wavelength strongly depends on the thicknesses of InAs wells and AlSb barriers, frequently in the range of $1-2 \mathrm{~nm}^{2}$ or even thinner, ${ }^{12}$ various thicknesses were investigated down to the thinnest possible InAs or AlSb layers while keeping perfect interfaces.

DFT calculations were performed in the Local Density Approximation (LDA) for the exchange and correlation energy using the Vienna Ab-initio Simulation Package (VASP). ${ }^{13-15}$ The structural properties of semiconductors calculated by the LDA are in better agreement with experimental values than using the Generalized Gradient Approximation. ${ }^{16}$ Electron-ion interactions were described using pseudopotentials constructed with the Projector Augmented Wave (PAW) method ${ }^{17}$ with the $5 s^{2}$ and $5 p^{1}$ valence electrons for In, $4 s^{2}$ and $4 \mathrm{p}^{3}$ for As, $3 \mathrm{~s}^{2}$ and $3 \mathrm{p}^{1}$ for $\mathrm{Al}$, and $5 \mathrm{~s}^{2}$ and $5 \mathrm{p}^{3}$ for $\mathrm{Sb}$. As this study focused on the structural properties, semi-core $d$ states of these elements were not included. All boundary conditions were periodic. Energy convergence was achieved by using cutoff energies of $700 \mathrm{eV}$, a $13 \times 13 \times 13$ (for bulk materials) or $13 \times 13 \times 1$ (for heterostructures) MonkhorstPack $^{18}$ mesh of $\mathrm{k}$ points and a Methfessel-Paxton method ${ }^{19}$ with a smearing of $0.01 \mathrm{eV}$.

Atomic positions were optimized by using conjugate gradient algorithm until the forces on each atom were lower than $0.02 \mathrm{eV} / \AA$. The cell sizes were fixed accordingly to the desired in-plane strain in the (001) plane and optimized by energy minimization in the [001] direction. 
Given the $\left(\overrightarrow{e_{1}}, \overrightarrow{e_{2}}, \overrightarrow{e_{3}}\right)$ axis system defined here by the [100], [010], and [001] directions, strain tensor components $\varepsilon_{i j}$ are deduced from the definition of the Lagrange strain tensor $\overline{\bar{\varepsilon}}$

$$
\varepsilon_{i j}=\frac{1}{2}\left(\frac{\partial u_{j}}{\partial x_{i}}+\frac{\partial u_{i}}{\partial x_{j}}+\frac{\partial u_{k}}{\partial x_{i}} \frac{\partial u_{k}}{\partial x_{j}}\right)
$$

considering that a point at position $\vec{x}=\left(x_{1}, x_{2}, x_{3}\right)$ in the reference state of a system is displaced by a quantity $\vec{u}(\vec{x})$ after the system deformation. Eq. (1) relies on the hypothesis of a continuum description of matter.

Here, the stress (strain) is biaxial (tetragonal) and homogeneous. We define the in-plane $\varepsilon_{/ /}$and out-of-plane $\varepsilon_{\perp}$ strains as $\varepsilon_{/ /}=\varepsilon_{11}=\varepsilon_{22}$ and $\varepsilon_{\perp}=\varepsilon_{33}$. Considering $a_{0}$ the lattice parameter of the reference state, here the material equilibrium state, and $a_{i i}$ the lattice parameter in the $i$ direction after deformation, the normal strain components come from (1) as

$$
\varepsilon_{i i}=\frac{\left(a_{i i}\right)^{2}-\left(a_{0}\right)^{2}}{2\left(a_{0}\right)^{2}} .
$$

Stress tensors $\overline{\bar{\sigma}}$ are calculated from the variation of the elastic energy, $d U_{\text {elast }}$, as a function of the strain variation $\delta \varepsilon_{i j}$. For an infinitesimal deformation

$$
d U_{\text {elast }}=\int_{V_{0}} \sigma_{i j} \delta \varepsilon_{i j} d x_{1} d x_{2} d x_{3}
$$

with $\mathrm{V}_{0}$ the volume of the reference state, $\sigma_{i j}$ the stress components defined by

$$
\sigma_{i j}=\frac{\partial u_{\text {elast }}}{\partial \varepsilon_{i j}}
$$

$u_{\text {elast }}$ the volume elastic energy.

The above definitions are general and do not suppose any relation between strain and stress tensors. The linear elasticity theory ${ }^{1}$ relies on the linear relationship (Hooke's law)

$$
\overline{\bar{\sigma}}=\mathrm{C} \overline{\bar{\varepsilon}},
$$

where $\mathrm{C}$ is the stiffness tensor describing the elastic properties of the material. In the case of interest here, all materials are cubic: their stiffness tensors are composed of 3 independent elastic constants, $\mathrm{C}_{11}, \mathrm{C}_{12}$, and $\mathrm{C}_{44}$ using the Voigt notation. The biaxial stress $\sigma_{/ /}=\sigma_{11}=\sigma_{22}, \varepsilon_{\perp}$ and $u_{\text {elast }}$ are thus related to $\varepsilon_{/ /}$by the well-known relationships

$$
\sigma_{/ /}=\mathrm{C}^{\prime} \varepsilon_{/ /}, \quad \varepsilon_{\perp}=-2 \frac{\mathrm{C}_{12}}{\mathrm{C}_{11}} \varepsilon_{/ /}, \quad u_{\text {elast }}=\mathrm{C}^{\prime} \varepsilon_{/ /}^{2},
$$

where

$$
\mathrm{C}^{\prime}=\mathrm{C}_{11}+\mathrm{C}_{12}-2 \frac{\mathrm{C}_{12}^{2}}{\mathrm{C}_{11}}
$$

If the material is coherently grown on a substrate of lattice parameter $a_{s u b}, \varepsilon_{/ /}$and the lattice parameters $a_{i i}$ can be written as function of the misfit $f$

$$
\varepsilon_{/ /}=-\frac{f(2+f)}{2(1+f)^{2}},
$$

where

$$
f=\frac{a_{0}-a_{\text {sub }}}{a_{\text {sub }}},
$$

and

$$
a_{11}=a_{22}=a_{\text {sub }}
$$

$$
a_{33}=a_{0} \sqrt{1+2 \frac{\mathrm{C}_{12} f(2+f)}{\mathrm{C}_{11}} \frac{f(1+f)^{2}}{(1+}} .
$$

(Note that for $f \ll 1$, a first-order development of (8) and (9) leads to

$$
\varepsilon_{/ /}=-f, \quad \varepsilon_{\perp}=2 \frac{\mathrm{C}_{12}}{\mathrm{C}_{11}} f
$$

and

$$
a_{33}=a_{0}\left(1+2 \frac{\mathrm{C}_{12}}{\mathrm{C}_{11}} f\right) .
$$

These simplified expressions are the same as usually established by omitting the non-linear contribution from Eq. (1), i.e., in the frame of small displacement conditions. However, in the case of high strain as in this study, non-linear contributions in Eq. (1) cannot be neglected ${ }^{20}$.)

Lattice parameters $a_{0}$ of InAs, AlSb, AlAs, and InSb bulk materials were computed from the fit of the total energy curves as a function of the unit cell volume by using the Murnaghan equation. ${ }^{21}$ Elastic stiffness constants $C_{i j}$ were determined from the response of the lattice to six finite distortions. $^{22}$ The results are reported in Table I together with experimental data. ${ }^{23}$ A good agreement is observed: errors on elastic constants are smaller than $10 \%$ and of the same order of magnitude as in previous DFT studies. ${ }^{24,25}$ In the following, all comparisons between DFT and linear elasticity will be based on the DFT calculated $a_{0}$ and $\mathrm{C}_{\mathrm{ij}}$ reported in Table I.

Elastic responses of bulk InAs, AlSb, AlAs, and InSb subjected to an in-plane biaxial stress were first investigated. The in-plane strain $\varepsilon_{/ /}$was imposed from $-7.5 \%$ to $7.5 \%$. Elastic energies computed by DFT are reported in Fig. 1(a) (dots) and compared to Eq. (6) (full lines). A significant deviation with the linear elasticity theory appears for inplane strains $\left|\varepsilon_{/ /}\right|>2.5 \%$ for all binaries. This limit value of $2.5 \%$ agrees with previous results reported for InAs. ${ }^{11}$ The

TABLE I. Lattice parameters $a_{0}$ and elastic stiffness constants $\mathrm{C}_{\mathrm{ij}}$ of bulk InAs, AlSb, AlAs, and InSb calculated by DFT (this work) and experimental data from Ref. 23. $\mathrm{C}^{\prime}$ is the first-order coefficient of the polynomial $\sigma_{/ /}$as a function of $\varepsilon_{/ /}$as calculated from (7). The coefficients $\alpha$ and $\beta$ of the nonlinear correction of $\sigma_{/ /}$are deduced from DFT.

\begin{tabular}{lccccc}
\hline \hline Bulk material & & AlAs & AlSb & InAs & InSb \\
\hline$a_{0}(\mathrm{~nm})$ & Exp. & 0.5660 & 0.6135 & 0.6058 & 0.6479 \\
& This work & 0.5638 & 0.6120 & 0.6059 & 0.6470 \\
misfit $f$ & Exp. & $-6.57 \%$ & $1.27 \%$ & $0 \%$ & $6.95 \%$ \\
& This work & $-6.95 \%$ & $1.01 \%$ & $0 \%$ & $6.78 \%$ \\
$\mathrm{C}_{11}(\mathrm{GPa})$ & Exp. & 125.48 & 87.79 & 84.40 & 66.66 \\
& This work & 112.36 & 83.97 & 84.58 & 65.87 \\
$\mathrm{C}_{12}(\mathrm{GPa})$ & Exp. & 53.54 & 43.51 & 46.40 & 36.41 \\
& This work & 57.07 & 42.71 & 49.39 & 36.93 \\
$\mathrm{C}_{44}(\mathrm{GPa})$ & Exp. & 54.39 & 40.77 & 39.60 & 30.20 \\
& This work & 53.16 & 38.10 & 36.91 & 29.02 \\
$\mathrm{C}^{\prime}(\mathrm{GPa})$ & From (7) & 111.45 & 83.23 & 76.29 & 61.39 \\
$\alpha$ & & 4.98 & 5.03 & 5.08 & 5.08 \\
$\beta$ & & 13.68 & 13.17 & 11.67 & 12.04 \\
\hline \hline
\end{tabular}



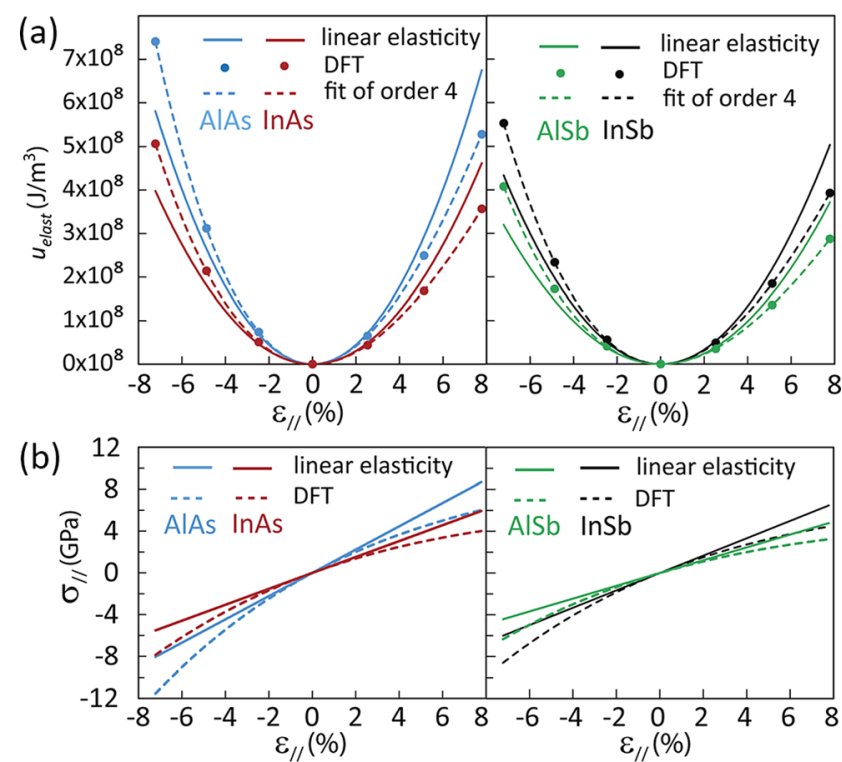

FIG. 1. (a) Volume elastic energy $u_{\text {elast }}$ calculated by linear elasticity (full lines), DFT (dots), and fit of the DFT results by a polynomial of order 4 (dashed lines) for bulk AlAs, InAs, AlSb, and InSb. (b) In-plane stress $\sigma_{/ /}$ calculated by linear elasticity from (6) (full lines) and by DFT from (11) (dashed lines), i.e., derivative of the fit of $u_{\text {elast }}$.

$u_{\text {elast }}$ curves as a function of $\varepsilon_{/ /}$were fitted by a polynomial of order 4 (dashed lines in Fig. 1(a)) where we imposed that the second-order coefficient $C^{\prime}$ is that calculated in the frame of the linear elasticity theory (Eq. (7)). Following Eq. (4), the in-plane stress components $\sigma / /$ were deduced from the derivation of this polynomial and can thus be written as

$$
\sigma_{/ /}=C^{\prime} \varepsilon_{/ /}\left(1-\alpha \varepsilon_{/ /}+\beta \varepsilon_{/ /}^{2}\right)
$$

Fig. 1(b) reports $\sigma_{/ /}$predicted by the linear elasticity (full lines) and calculated from DFT according to Eq. (11) (dashed lines). The coefficients $\alpha$ and $\beta$ given by the fits are reported in Table I. They express the dissymmetry between tension and compression in the deviation to the linear elasticity. The strain $1 / \alpha$ characterizes this deviation and is $20 \%$ for all studied binaries.

Fig. 2 reports $\varepsilon_{\perp}$ as a function of $\varepsilon_{/ /}$. For all materials, $\varepsilon_{\perp}$ computed by DFT (dots) is proportional to $\varepsilon_{/ /}$in the whole range of studied strain and is in very good agreement with the value predicted by linear elasticity theory using Eq. (6) (full line): the largest relative differences 0.04 and 0.06
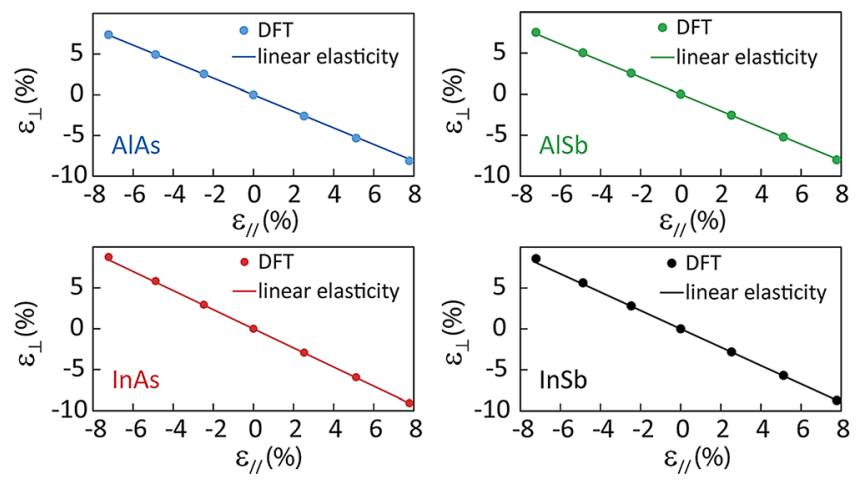

FIG. 2. Out-of-plane strain $\varepsilon_{\perp}$ as a function of the in-plane strain $\varepsilon_{/ /}$computed by DFT (dots) and prediction of the linear elasticity theory Eq. (6) (full lines) for bulk AlAs, InAs, AlSb, and InSb. are observed for $\varepsilon_{/ /}=-7.5 \%$ in InAs and $\mathrm{InSb}$, respectively, while in all other cases the relative difference is less than 0.03 . As already reported in a previous DFT study of InAs, ${ }^{24}$ the ratio $2\left(\mathrm{C}_{12} / \mathrm{C}_{11}\right)$ can thus be used as a tool to calculate $\varepsilon_{\perp} / \varepsilon_{/ /}$even for high $\left|\varepsilon_{/ /}\right|$(approx. $7.5 \%$ ).

[InAs/AlSb] periodic heterostructures were then investigated. To reproduce the epitaxial configuration, the in-plane lattice parameter was fixed at the bulk InAs one $\left(a_{11}=a_{22}\right.$ $\left.=a_{\text {sub }}=0.6059 \mathrm{~nm}\right)$. The configuration with two AlAs-like perfect interfaces was examined in a simulation cell composed of $m+1$ and $m$ successive planes of As and In atoms in the [001] direction followed by $n+1$ and $n$ ones of $\mathrm{Al}$ and $\mathrm{Sb}$ ( $m+n$ being odd to insure the periodicity); $m$ and/or $n$ varying from 1 to 9 were investigated.

Fig. 3(a) reports a schematic representation of the case $(m=8, n=5)$ and the distance $d$ between two adjacent atomic layers as a function of the successive atomic layers along [001], computed from DFT (blue dots) and from the linear elasticity theory (green squares). Note that for a bulk, $d$ would simply be the quarter of $a_{0}$ in the reference state or of $a_{33}$ in the strained state (Eq. (9)). In the InAs region, $d$ computed by DFT is thus compared with its value in
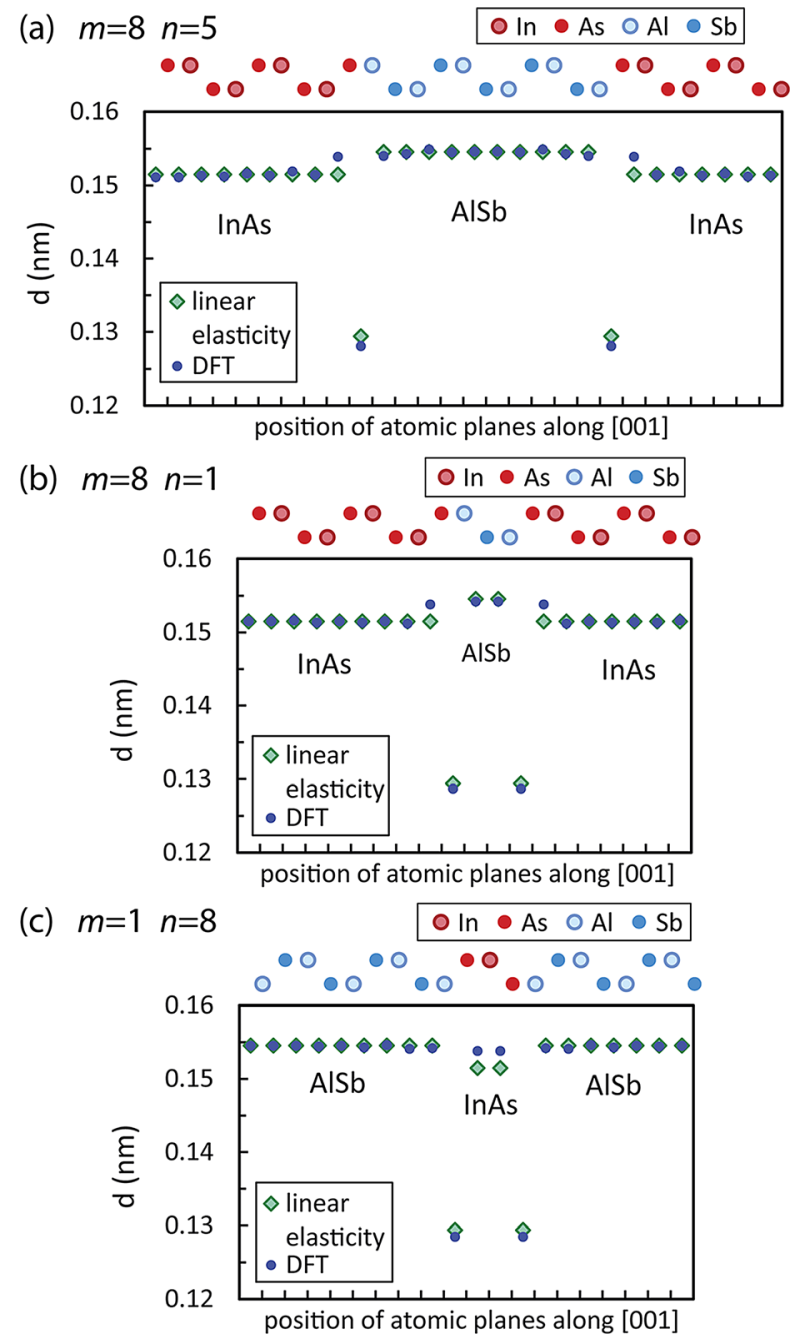

FIG. 3. Schematic projections on a $\{110\}$ plane of periodic [InAs/AlSb] multilayers and interplane distances $d$ as a function of the position of the atomic layers in the [001] direction. One period consists of $m+1$ and $m$ planes of As and In atoms and $n+1$ and $n$ planes of $\mathrm{Al}$ and $\mathrm{Sb}$. It includes two AlAs interfaces. (a) $m=8, n=5$. (b) $m=8, n=1$. (c) $m=1, n=8$. 
unstrained bulk InAs, while in the AlSb regions, it is compared with the values predicted by the linear elasticity in bulk AlSb subjected to the same in-plane strain as in the heterostructure. At the interface position, $d$ is compared to the value predicted by the linear elasticity theory in bulk AlAs subjected to the same in-plane strain as in the heterostructure $(7.75 \%)$.

As expected, the linear elasticity provides a very good estimation of $d$ in the InAs and AlSb regions. The maximum relative error is $0.3 \%$, except for the first interplane distance in InAs for which $d$ given by linear elasticity $(0.1515 \mathrm{~nm})$ is $1.6 \%$ underestimated compared to the DFT value $(0.1540 \mathrm{~nm})$. At the interfaces, though the comparison with strained bulk AlAs is questionable, the prediction of the elasticity theory from Eq. (9) surprisingly gives a good estimation of $d$ $(0.1294 \mathrm{~nm})$ with only $\sim 0.9 \%$ overestimation compared to the value calculated by DFT $(0.1283 \mathrm{~nm})$. Applying Eq. (2), the corresponding $\varepsilon_{\perp}$ strain is $-7.9 \%$ ( $d$ from linear elasticity) and $-8.6 \%$ ( $d$ from DFT). The difference between these two values is smaller than the precision of experimental measures done at interfaces by analyzing atomic resolved (Scanning)-TEM images. ${ }^{4}$ Heterostructures with larger or smaller values of $m$ and/or $n$ presented similar results (not shown).

For the thinnest possible AlSb layer, the two interfaces are only separated by one atomic Sb layer $(n=1)$; one could expect to alter the interplane distances at interfaces. Fig. 3(b) reports the distances $d$ for $m=8$ and $n=1$. Surprisingly, the same results as for thick AlSb layers are observed at interfaces (the DFT value equals $0.1287 \mathrm{~nm}$ ). The same conclusions also arise when the InAs layer is reduced to $m=1$ (interfaces separated by a single atomic In layer). The case $m=1$ and $n=8$ is displayed in Fig. 3(c).

For perfect InSb-type interfaces, $p+1$ and $p$ successive planes of In and As atoms followed by $q+1$ and $q$ ones of $\mathrm{Sb}$ and $\mathrm{Al}(p+q$ being odd) were simulated; $p$ and/or $q$ from 1 to 9 were investigated. Fig. 4(a) displays the case $(p=8$, $q=5$ ); the interplane distance $d$ is reported as previously, except at the interface where the predicted value is calculated in bulk InSb subjected to the same in-plane strain as in the heterostructure $(-6.15 \%)$. The linear elasticity theory provides good estimation of $d$ in InAs and AlSb regions with a maximum relative error of $0.5 \%$, except for the first interplane distance in $\mathrm{AlSb}$ for which $d$ given by linear elasticity $(0.1545 \mathrm{~nm})$ is $1.0 \%$ overestimated compared to the DFT value $(0.1530 \mathrm{~nm})$.

At the interface, again the prediction of the linear elasticity theory $(d=0.1725 \mathrm{~nm})$ provides an unexpected good estimation, only $1.1 \%$ smaller than the DFT calculated value $(0.1745 \mathrm{~nm})$. The corresponding $\varepsilon_{\perp}$ calculated with (2) from $d$ is $6.9 \%$ (linear elasticity) and $8.2 \%$ (DFT). Similarly to Al-As interfaces, the precision of (scanning)-TEM images analysis ${ }^{4}$ is not sufficient to capture the difference between these two strain values. No significant change occurs when AlSb is reduced to the smallest thickness, i.e., when $p \geq 4$ and $q=1$ as illustrated in Fig. 4(b) for $p=8, q=1$. Similar results occur for $p=1$ and $q \geq 4$ (reduction of InAs layer) as seen in Fig. 4(c).

To conclude, in bulk InAs, AlAs, InSb, and AlSb biaxially stressed in a (001) plane, DFT calculations showed a significant deviation to the theory of linear elasticity for

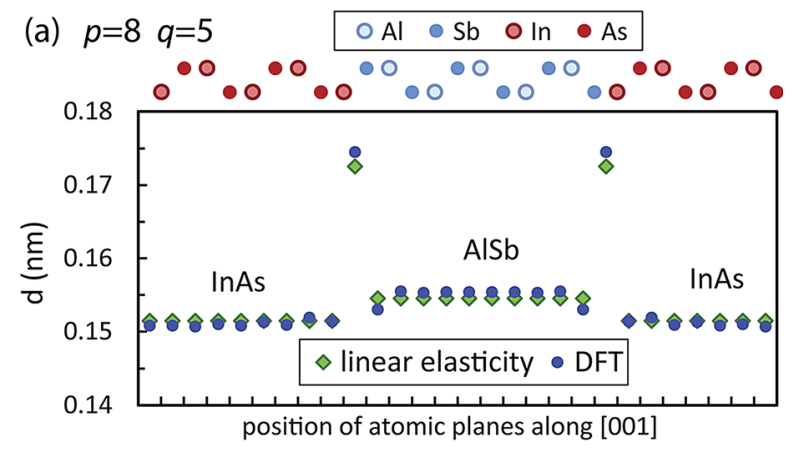

(b) $p=8 \quad q=1$

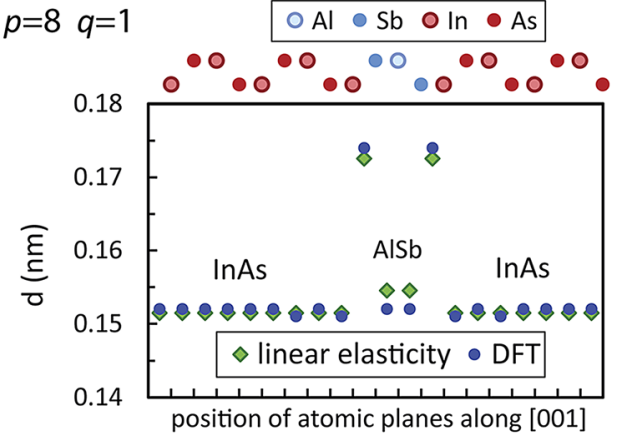

(c) $p=1 \quad q=8$

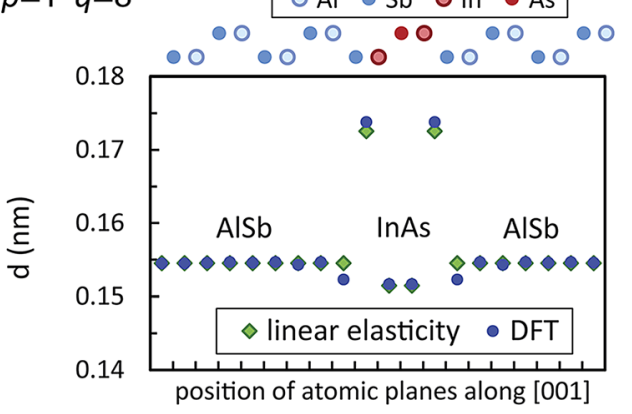

FIG. 4. Schematic projections on a $\{110\}$ plane of periodic [InAs/AlSb] multilayers and interplane distances $d$ as a function of the position of the atomic layers in the [001] direction. One period consists of $p+1$ and $p$ planes of In and As atoms and $q+1$ and $q$ planes of $\mathrm{Sb}$ and Al. It includes two InSb interfaces. (a) $p=8, q=5$. (b) $p=8, q=1$. (c) $p=1, q=8$.

strain above $2.5 \%$ (in absolute value). Correction terms for the biaxial stress, similar for the four studied binaries, were deduced by fitting the elastic energy calculated by DFT. Regarding the perpendicular strain, the prediction by linear elasticity was in very good agreement with the DFT calculations, even for large strain up to at least $7.5 \%$ (in absolute value), and can thus be used as a tool to calculate large strain in this configuration.

Finally, in the [InAs/AlSb] heterostructures, the structures of perfect $\mathrm{Al}-\mathrm{As}(\mathrm{In}-\mathrm{Sb})$ interfaces predicted by applying the linear elasticity as in bulk AlAs ( $\mathrm{InSb}$ ) were in a surprisingly good agreement with DFT calculations. Strains only differed of a few \%. The reduction of the thickness of InAs or AlSb layer did not change these conclusions, even for ultra-thin layers consisting in one plane of the group III element surrounded by two planes of the group V element, or the reverse situation.

This work was supported by the French research national agency project NAIADE (Grant No. ANR-11-BS10-017). DFT calculations were performed on the EOS supercomputer of CALMIP (Calcul en Midi-Pyrénées, Grant No. p0933). 
${ }^{1}$ L. D. Landau, L. P. Pitaevskii, A. M. Kosevich, and E. M. Lifshitz, Theory of Elasticity (Elsevier, 1984).

${ }^{2}$ O. Cathabard, R. Teissier, J. Devenson, J. C. Moreno, and A. N. Baranov, Appl. Phys. Lett. 96, 141110 (2010).

${ }^{3}$ F. Raouafi, R. Benchamekh, M. O. Nestoklon, J.-M. Jancu, and P. Voisin, J. Phys.: Condens. Matter 28, 045001 (2016).

${ }^{4}$ M. Vallet, Y. Claveau, B. Warot-Fonrose, C. Gatel, J. Nicolaï, N. Combe, C. Magen, R. Teissier, A. N. Baranov, and A. Ponchet, Appl. Phys. Lett. 108, 211908 (2016).

${ }^{5}$ J. Nicolaï, B. Warot-Fonrose, C. Gatel, R. Teissier, A. N. Baranov, C. Magen, and A. Ponchet, J. Appl. Phys. 118, 035305 (2015).

${ }^{6}$ K. Mahalingam, H. J. Haugan, G. J. Brown, and K. G. Eyink, Ultramicroscopy 127, 70 (2013).

${ }^{7}$ B. Bonef, B. Haas, J. L. Rouviere, R. Andre, C. Bougerol, A. Grenier, P. H. Jouneau, and J. M. Zuo, J. Microsc. 262, 178 (2016).

${ }^{8}$ M. de la Mata, C. Magen, P. Caroff, and J. Arbiol, Nano Lett. 14, 6614 (2014).

${ }^{9}$ C. Y. Wen, M. C. Reuter, D. Su, E. A. Stach, and F. M. Ross, Nano Lett. 15, 1654 (2015).

${ }^{10}$ A. Patra, J. K. Panda, A. Roy, M. Gemmi, J. David, D. Ercolani, and L. Sorba, Appl. Phys. Lett. 107, 093103 (2015).
${ }^{11}$ T. Hammerschmidt, P. Kratzer, and M. Scheffler, Phys. Rev. B 75, 235328 (2007).

${ }^{12}$ M. Bahriz, G. Lollia, P. Laffaille, A. N. Baranov, and R. Teissier, Electron. Lett. 49, 1238 (2013).

${ }^{13}$ G. Kresse and J. Hafner, Phys. Rev. B 47, 558 (1993).

${ }^{14}$ G. Kresse and J. Furthmüller, Phys. Rev. B 54, 11169 (1996).

${ }^{15}$ G. Kresse and J. Furthmüller, Comput. Mater. Sci. 6, 15 (1996).

${ }^{16}$ C. Filippi, D. J. Singh, and C. J. Umrigar, Phys. Rev. B 50, 14947 (1994).

${ }^{17}$ P. E. Blöchl, Phys. Rev. B 50, 17953 (1994).

${ }^{18}$ H. J. Monkhorst and J. D. Pack, Phys. Rev. B 13(12), 5188 (1976).

${ }^{19}$ M. Methfessel and A. T. Paxton, Phys. Rev. B 40(6), 3616 (1989).

${ }^{20}$ A. F. Bowers, Applied Mechanics of Solids (http://solidmechanics.org and CRC Press, 2010).

${ }^{21}$ F. D. Murnaghan, Proc. Natl. Acad. Sci. USA 30, 244 (1944).

${ }^{22}$ Y. Le Page and P. Saxe, Phys. Rev. B 65, 104104 (2002).

${ }^{23}$ V. Swaminathan and A. T. Macrander, Materials Aspects of GaAs and InP Based Structures (Prentice-Hall, Inc., Englewood Cliffs, New Jersey, 1991).

${ }^{24}$ L. Pedesseau, J. Even, A. Bondi, W. Guo, S. Richard, H. Folliot, C. Labbe, C. Cornet, O. Dehaese, A. Le Corre, O. Durand, and S. Loualiche, J. Phys. D: Appl. Phys. 41, 165505 (2008).

${ }^{25}$ S. Q. Wang and H. Q. Ye, Phys. Status Solidi B 240, 45 (2003). 